\title{
Circumstellar Interaction Around Supernovae
}

\author{
Roger A. Chevalier \\ Department of Astronomy, University of Virginia, P.O. Box 3818, \\ Charlottesville, VA 22903, USA
}

\begin{abstract}
Circumstellar interaction has been observed around all types of massive star supernovae, especially at radio and X-ray wavelengths. The interaction shells in Type Ib/c supernovae appear to be moving rapidly, although SN 1998bw remains the only case for which the evidence points to relativistic expansion. Type IIP supernovae have relatively low mass loss rates, consistent with moderate mass single stars at the ends of their lives. Type IIb and some IIL and IIn supernovae can have strong mass loss leading up to the explosion, which occurs in a star with little $\mathrm{H}$ on it. The mass loss may be driven by the luminosity of the late burning stages.
\end{abstract}

\section{Introduction}

During their lifetimes, massive stars undergo mass loss during various evolutionary phases. When they finally end their lives as supernovae, the interaction reveals the mass loss processes leading up to the explosion. and the structure of the exploding star. Recent multiwavelength observations have revealed many facets of the interaction and we now have observations showing circumstellar interaction around all types of massive star supernovae. Circumstellar interaction can be related to late massive star evolution and to the physical processes in the dense gas interaction. Analysis of the radio and X-ray emission from supernovae yields the energy in high velocity ejecta and can be used to set limits on the amount of relativistic ejecta; the relation of supernovae to gamma-ray bursts can thus be explored.

A recent review of circumstellar interaction with an emphasis on the relevant physical processes is in 9. Here, I briefly review recent developments, emphasizing the relation of circumstellar interaction to the various supernova types. In $\S 2$, I discuss the wide range of multiwavelength observations of circumstellar interaction that is now available. Interaction around the various types of supernovae is discussed in $\S 3$. Conclusions are in $\S 4$.

\section{Multiwavelength observations of interaction}

One of the most sensitive ways of finding or observing supernovae with circumstellar interaction is through their nonthermal, radio synchrotron emission. The radio light curves of extragalactic supernovae show an early absorbed phase, so that there is a rise to a maximum followed by an approximately power law decline [58]. Fig. 1 shows the relationship between the observed peak luminosity 


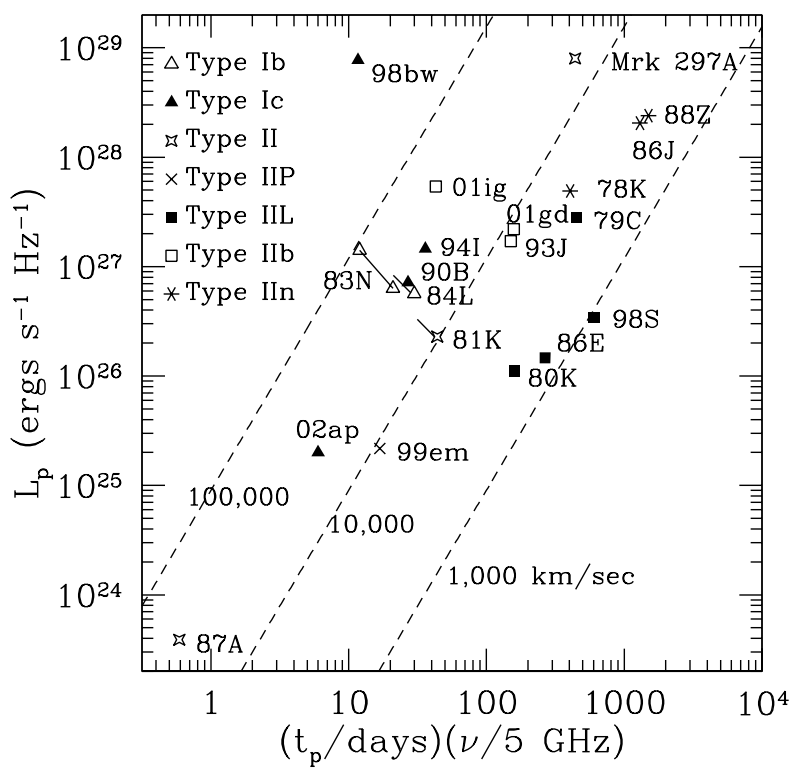

Fig. 1. Peak luminosity and corresponding epoch for the well-observed radio supernovae. The dashed lines give curves of constant expansion velocity, assuming synchrotron self-absorption (see [7]).

and the age at peak luminosity (from [7, with updates). Recent detections are SN 2001ig [48, SN 2001gd [52, and SN 2002ap [4]. All types of massive star supernovae are represented, including Type Ib/c which are thought to have lost their hydrogen envelopes before the explosion, Type IIb which have lost almost all their hydrogen envelopes before the explosion, Type IIn with narrow line optical emission, Type IIL with linear optical light curves, Type IIP with plateau light curves, and the peculiar SN 1987A. Interestingly, the different types of supernovae populate different parts of this diagram. Although the numbers are still small, some trends are showing up in the interaction properties. The Type $\mathrm{Ib} / \mathrm{c}$ supernovae peak early and have a range of luminosity, while the Type IIL and Type IIn SNe peak late and also have a range of luminosity. The Type IIb are intermediate between these and the one Type IIP is of low luminosity. The early peak of the Type $\mathrm{Ib} / \mathrm{c}$ supernovae is an indication that their radio emitting regions expand relatively rapidly $[7$.

The other wavelength region where young supernova remnants are broadly observed is X-rays. Fig. 2 shows the relation between the X-ray and radio luminosity of young remnants. Here, the radio luminosity is estimated from $\nu L_{\nu}$ at $5 \mathrm{GHz}$. X-ray emission has been detected from the full range of massive star supernovae, and there is a clear correlation of the X-ray and radio emission. The X-ray luminosities, primarily from [26], are not all in the same X-ray band, but 


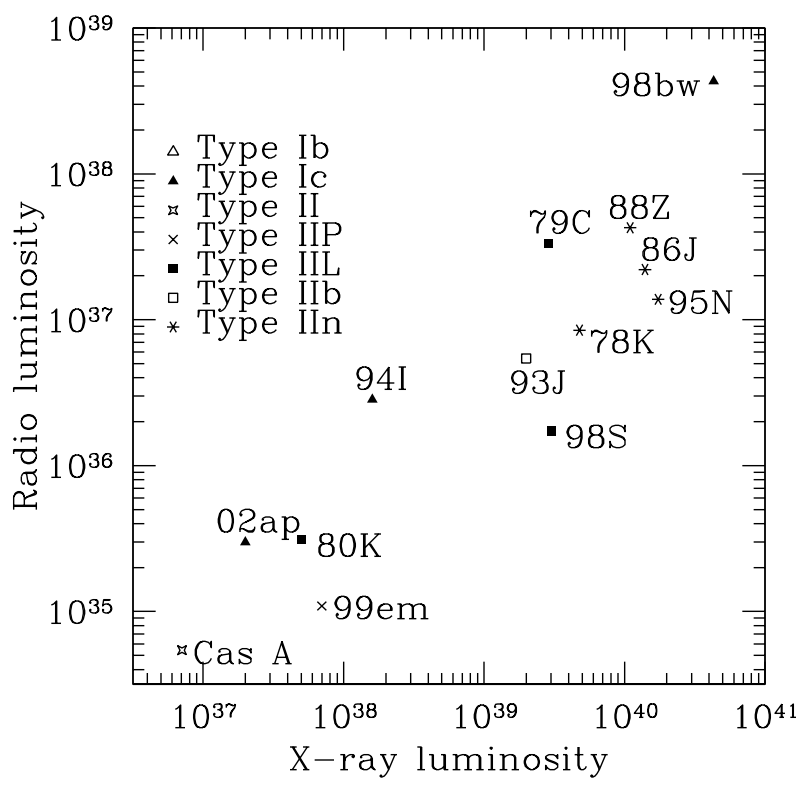

Fig. 2. Radio luminosity vs. X-ray luminosity of supernovae, both in $\operatorname{ergs} \mathrm{s}^{-1}$. The $\mathrm{X}$-ray data are primarily from [26].

this should not have a large effect on the correlation. X-ray line emission has been detected in a number of cases (SN 1987A [43], SN 1986J 24], SN 1993J [54], SN 1998S [46], Cas A), showing that the emission is thermal, at least in these cases. The correlation of the X-ray and radio emission shows that both of these give a measure of the strength of circumstellar interaction, even though they involve different radiation mechanisms. Radio emission provides a good indicator of whether a particular event is suitable for observation with the space missions Chandra and XMM.

At UVOIR (ultraviolet/optical/infrared) wavelengths, circumstellar interaction is generally not observed in Type $\mathrm{Ib} / \mathrm{c}$ and Type IIP supernovae, but is observed in the spectra of Type IIL, IIb, and IIn supernovae. The reason appears to be the relatively low densities and high velocities present in Type $\mathrm{Ib} / \mathrm{c}$ and Type IIP supernovae, so that radiative shock waves are not present; in addition, the X-ray luminosities are not sufficient to illuminate the expanding ejecta to observable levels. In the cases with stronger circumstellar interaction, UVOIR emission is potentially observable from shocked, cooled gas, from freely expanding ejecta, and from unshocked circumstellar gas. In the case of SN 1995N, all of these components have been observed [21]; ultraviolet $H S T$ observations are especially useful for observing the ejecta.

Strong infrared emission, presumably from dust, has been observed from the Type IIL supernovae SN 1979C and 1980K 13 and, recently, from a number 
of Type IIn supernovae 1512 23. The preferred explanation has been emission from radiatively heated circumstellar dust. Another possible source of emission is collisionally heated dust in the hot, shocked gas, as is observed in a number of Galactic supernova remnants, including Cas A [14]. The high observed luminosity implies that the source is not dust formed in the freely expanding ejecta.

\section{Circumstellar interaction and supernova types}

\subsection{Type Ib/c supernovae and "hypernovae"}

The best observed Type Ic supernova was SN 1994I in M51, which had a rapid decline after maximum light and can be interpreted as an explosion with an ejecta mass of only $0.9 M_{\odot} 3062$. It is the only normal Type Ic supernova to have been detected in X-rays [2729]. Chevalier [7] applied a synchrotron self-absorption model to the available radio data on SN 1994I and found an implied shock radius of about $1.2 \times 10^{16} \mathrm{~cm}$ at an age of 36 days, giving a shock velocity of $39,000 \mathrm{~km} \mathrm{~s}^{-1}$. Application of the asymptotic power law density profile of 40 ( $\left(\rho \propto r^{-10.1}\right)$ yields an outer shock radius $R=1.7 \times$ $10^{16}\left(M / M_{\odot}\right)^{-0.32}\left(E / 10^{51} \mathrm{ergs}\right)^{0.44}\left(\dot{M}_{-5} / v_{w 3}\right)^{-0.12} \mathrm{~cm}$, where $M$ is the ejecta mass, $E$ is the explosion energy, $\dot{M}_{-5}=\dot{M} / 10^{-5} M_{\odot} \mathrm{yr}^{-1}$ is the mass loss rate, and $v_{w 3}=v_{w} / 10^{3} \mathrm{~km} \mathrm{~s}^{-1}$ is the wind velocity 4. The approximate agreement of the hydrodynamic model with the radio radius may be improved by using a more accurate density model for the exploded star, because the density profile is not fully in the asymptotic power law regime for such a small mass star. Immler et al. 29] have used Chandra observations of SN 1994I to deduce a presupernova mass loss rate of $1 \times 10^{-5} M_{\odot} \mathrm{yr}^{-1}\left(v_{w} / 10 \mathrm{~km} \mathrm{~s}^{-1}\right)$. For a Wolf-Rayet star wind velocity $v_{w} \approx 10^{3} \mathrm{~km} \mathrm{~s}^{-1}$, the inferred mass loss rate of $1 \times 10^{-3} M_{\odot} \mathrm{yr}^{-1}$ is surprisingly high. However, Immler et al. 29] used a model for the interaction from Chevalier [6], which was developed before the recognition of Type Ib/c supernovae as a separate type and was based on a Type Ia interpretation. If the high mass loss rate holds up in a more detailed model, it may be evidence for mass loss from a companion star, as has previously been suggested based on radio observations of Type $\mathrm{Ib} / \mathrm{c}$ supernovae [56].

Much attention has recently been focused on Type Ic supernovae because of the occurrence of SN 1998bw, apparently associated with the gamma-ray burst GRB 980425 [22. The strong radio emission from the source at early times (Fig. 1) implied the presence of relativistic motions, further supporting the GRB connection [33 35. SN 1998bw also had an unusual optical spectrum, with velocities in lines extending out to $\sim 60,000 \mathrm{~km} \mathrm{~s}^{-1}[22$. The interpretation of the supernova light curve and spectra led to an explosion energy estimate of $(2-4) \times 10^{52}$ ergs, extraordinarily high for a supernova 3161. Other Type Ic supernovae have been discovered with broad lines in their spectra, including SN 1997ef, SN 2002ap, and SN 2002bl. Because of the high energies inferred for these events, Mazzali et al. 42 and others have called these objects hypernovae.

Outside of SN 1998bw, broad-lined Type Ic supernovae have not been directly connected with GRBs. In the case of SN 2002ap, there are limits on any corre- 
sponding GRB 25. Despite its broad line spectrum, SN 2002ap was a weaker radio source than the normal SN 1994I (Fig. 1; 4 ). Its position in Fig. 1 indicates a higher $M_{e j}$ or lower $E$ than SN 1994I, plus a lower circumstellar density. The model of Mazzali et al. [42] has $M_{e j}=2.4 M_{\odot}$, which can account for part of the difference, but there is no evidence for the high energy $\left(4 \times 10^{51} \mathrm{ergs}\right)$ that they infer for the explosion. X-ray observations of SN 2002ap with XMM also showed a surprisingly low luminosity [4]. This emission is sensitive to the distribution of high velocity gas and thus sets additional constraints to those obtained from optical observations. Sutaria et al. 53] suggested that inverse Compton is the most likely X-ray emission mechanism because of the relatively soft spectrum of the source. Approximate modeling of the radio emission in 4 showed that the energy in high velocity ejecta is small. The claim of high energies in broad line SN Ic is based on modeling the broad optical absorption lines. However, the radio emission probably gives a more direct estimate of the energy in high velocity ejecta and the possible relation to GRBs. The broad line Type Ic SN $2002 \mathrm{bl}$ was also a much weaker radio source than SN 1998bw [3].

The combination of radio and X-ray observations with a hydrodynamic model for the exploded star that is consistent with the observed optical supernova emission should make it possible to estimate the mass loss density around the supernova progenitor. This information will be useful in determining the progenitor evolution leading to an explosion. Type Ib/c supernovae occur at higher rates than expected for single stars that have lost their envelopes and binary evolution is a likely factor (e.g., 59 ). The mass loss properties remain uncertain parameters for the presupernova evolution of these objects.

\subsection{Type IIb supernovae}

The Type IIb supernova SN 1993J was in M81 at a distance of only about 3.6 $\mathrm{Mpc}$ and has become the second best observed case of circumstellar interaction, after SN 1987A. The exploded star in this case is thought to be an initially $12-$ $15 M_{\odot}$ star that lost most of its envelope mass as a result of binary interaction 4560 .

VLBI imaging shows an approximately symmetric, but variable, shell of radio emission [2]. The ratio of shell thickness to radius is $\Delta R / R \sim 0.3$ [37, which is larger than expected in standard interaction models 5 . A possible explanation is that inhomogenities in the wind lead to a broader region. Although this specific situation has not been modeled, there is evidence that shock interaction with a clumpy medium gives rise to a broader, more turbulent shocked medium than would otherwise be present 32. Relatively large scale inhomogeneities may be related to the variability that has been observed in the radio structure [2] An examination of the optical line profiles of the supernova also gives an indication of inhomogeneity. Without inhomogeneities, the corrugation of the reverse shock wave is relatively small [10, which would produce a more box-like $\mathrm{H} \alpha$ line than was observed 39 .

There has been some confusion regarding the ambient density profile for SN 1993J. Assuming a form $\rho_{w} \propto r^{-s}$, both radio 57203844 and X-ray 28] studies 
have led to $s=1.5-1.7$. This is a surprising result because it implies a mass loss rate decreasing as a power law in time leading up to the supernova; Immler et al. 28] deduce a gradual reduction in $\dot{M}$ by $>10$ based on the X-ray light curve from ROSAT observations. However, the initial radio studies assumed that free-free absorption was the primary absorption process. Fransson \& Björnsson [19] showed that other physical processes, especially synchrotron self-absorption, are important for the radio curve and a detailed model yields $s=2$, which is the expected value close to a massive star. The X-ray result of Immler et al. 28 appears to be due to an interpretation of the slowly decaying X-ray flux in terms of an adiabatic shocked region. In fact, the soft X-ray emission in the ROSAT band is dominated by emission from the reverse shock, which we expect to be radiative 20. The X-ray spectra from $A S C A$ give further evidence for a cool shell formed downstream from a radiative shock [55. In the radiative case with $s=2$, the X-ray emission is expected to be relatively constant when it is not absorbed 8]. Swartz et al. 54 have obtained a Chandra spectrum of SN 1993J at an age of 7 years. It shows cool and hot components that can be identified with the reverse shock wave and the forward shock, respectively. The cool component itself requires two temperature components ( 0.35 and 1.01 $\mathrm{keV}$ ) to fit the spectrum. This may be an indication that the emission is from a radiatively cooling shock front.

\subsection{Type IIn/IIL supernovae}

The Type IIn and IIL designations are not necessarily distinct; for example, SN 1998S had a light curve that led to a IIL designation [36], but also had narrow lines, leading to a IIn designation [34. SN 1995N and SN 1998S are the best observed recent supernovae in the IIn category. There have been recent HST 21] and X-ray 1846 observations of these supernovae. They both have Xray luminosities in the range $10^{40}-10^{41} \mathrm{erg} \mathrm{s}^{-1}$, are strong radio emitters, and show narrow lines in their optical spectra. Both supernovae show evidence for the reverse shock front moving into heavy element rich gas. In the case of SN 1995N, the UV/optical spectra show evidence for emission from photoionized gas just inside the reverse shock front, including the OI $\lambda 7774$ recombination line, but there is no $\mathrm{H}$ emission associated with this component 21]. Chandra spectra for SN 1995N are not yet available, but summed Chandra spectra of SN 1998S show evidence for an overabundance of the Si group elements 46. Thus, although these supernovae were of Type II, with hydrogen in their spectra at early times, they developed evidence for a reverse shock front moving into enriched material within several years of the explosion. In addition, Leonard et al. 34 noted that the photospheric spectrum of SN 1998S at an age of 25 days resembled that of a Type Ic supernova, with absorption lines of OI $\lambda 7774$ and SiII $\lambda 6355$ and weak $\mathrm{H} \alpha$ emission. The implication is that strong mass loss just before the supernova almost entirely removed the hydrogen envelope.

Additional observations of SN 1998S have shown a number of interesting features. The evolution of the optical spectrum has been interpreted as indicating several regions of mass loss through which the supernova shock front is moving 
34 15. The supernova, which was discovered on 3 March, 1998, showed symmetric broad lines with FWZI (full width at zero intensity) of $\sim 20,000 \mathrm{~km} \mathrm{~s}^{-1}$ until 12 March, 1998. The sudden disappearance of the lines can be interpreted as due to the shock wave overtaking an inner dense region. Chugai [1] has recently interpreted the broad lines as due to scattering in a dense circumstellar medium out to $\sim 10^{15} \mathrm{~cm}$. This requires $\dot{M}_{-5} / v_{w 1} \gtrsim 300$ in this region. Leonard et al. 34. find little evidence for subsequent circumstellar interaction until an age of 108 days. The later X-ray and radio observations imply $\dot{M}_{-5} / v_{w 1} \sim(10-20)$ [46. An early infrared excess implies the presence of circumstellar dust [15, which places additional constraints on the circumstellar density and dust content.

Fassia et al. [16] observed narrow emission lines and inferred that the outer circumstellar medium had a velocity of $40-50 \mathrm{~km} \mathrm{~s}^{-1}$. From the time evolution and velocities, they inferred that the outer wind started at least 170 years ago, stopped about 20 years ago, and the inner dense wind may have started less than 9 years ago. The variability might be related to the mass loss variability observed in late type stars.

The structure of red supergiant winds is uncertain, because they are generally quite distant. However, red giant AGB (asymptotic giant branch) stars are known to have a semi-periodic shell structure, observed in scattered light (e.g., IRC +10216 , 41]) as well as in the HST images of several planetary nebulae (e.g., 1]). The characteristic time scale for the shells, several 100 years, is indicative of an instability in the acceleration mechanism for the wind. Similar mass loss processes are likely to occur in red supergiants; the mass loss characteristics of IRC +10216 are $v_{w}=14 \mathrm{~km} \mathrm{~s}^{-1}$ and $\dot{M}=2 \times 10^{-5} M_{\odot} \mathrm{yr}^{-1}$, which are comparable to values for red supergiants. The shell properties for IRC +10216 can be determined from observations of dust scattered light [41. The shells have a density contrast of a factor 3 over the rest of the wind and contain a substantial fraction of the total mass in the wind. If the supernova is expanding at $\sim 10^{4} \mathrm{~km} \mathrm{~s}^{-1}$, the interaction should lead to structure in the supernova light curves on a timescale of $\sim 100$ days.

It is also possible that the mass loss properties are determined by the late nuclear burning phases just before the explosion. When there is very little $\mathrm{H}$ envelope left on a star, the nuclear processes can play a role in the mass loss properties. Both SN 1995N and SN 1998S appear to have exploded at a time of strong mass loss when the $\mathrm{H}$ envelope was almost entirely lost. SN 1993J also exploded with a modest $\mathrm{H}$ envelope (few $0.1 M_{\odot}$ ); in this case, the small envelope mass has been attributed to binary interaction [4560. However, the mass loss from the envelope should then be especially strong when the star first becomes a red supergiant and is not very powerful at the time of the explosion. SN 1993J in fact had strong mass loss at the time of the explosion and in the cases of SN 1995N and SN 1998S, the mass loss was even stronger. These observations suggest the possibility that the heightened luminosity in the late burning stages plays a role in driving the strong mass loss that leads to the loss of the $\mathrm{H}$ envelope. 


\subsection{Type IIP supernovae}

There is little evidence for circumstellar interaction around Type IIP supernovae, but the evidence that we have indicates a low circumstellar density. Fig. 1 shows the relatively low radio luminosity of SN $1999 \mathrm{em}$. The X-ray luminosity of SN $1999 \mathrm{em}$ is correspondingly low (Fig. 2; 46]) and the X-ray luminosity of the Type IIP SN 1999gi is similarly low [50]. Approximate models for the X-ray emission lead to a mass loss rate of $(1-2) \times 10^{-6} M_{\odot} \mathrm{yr}^{-1}\left(v_{w} / 10 \mathrm{~km} \mathrm{~s}^{-1}\right)$.

Information on the initial mass of supernovae comes from studies of their stellar environments, especially with HST; such studies have been carried out for the Type IIP SNe 1999em and 1999gi [51. These studies indicate that these Type IIP supernovae may have come from $8-12 M_{\odot}$ stars. Type IIP supernovae are compatible with single star evolution and initial masses $\sim 8-15 M_{\odot}$ and relatively little mass loss during the evolution. This is consistent with the evolutionary models of Schaller et al. 49 who find that these stars end their lives as RSGs with $\dot{M} \sim 2 \times 10^{-6} M_{\odot} \mathrm{yr}^{-1}$, in agreement with the properties noted above.

\section{Conclusions}

Early circumstellar interaction gives rise to a number of interesting physical processes in dense media, including radiative $\sim 1,000 \mathrm{~km} \mathrm{~s}^{-1}$ shock waves, particle acceleration in fast shock waves, and the emission of infrared dust echoes. The conditions in circumstellar interaction approach those in quasars; in fact, some supernovae which are likely circumstellar interactors have spectra similar to Seyfert galaxies [17, but the physical situation is better understood for the supernova case. Analysis of the observations shows the mass loss processes leading to the supernova.

The observational support for these studies is promising. The Chandra and $X M M$-Newton X-ray missions have made the X-ray emission from nearby supernovae accessible. SOFIA and SIRTF should contribute to our understanding of infrared dust emission from supernovae. Together with ground-based observations, the detailed picture of supernova interaction with circumstellar matter should give considerable insight into the late evolution of massive stars.

I am grateful to Claes Fransson for collaboration and discussion of these topics. This research was supported in part by NASA grant NAG 5-8232 and grant GO-08648.03-A from the Space Telescope Science Institute, which is operated by the Association of Universities for Research in Astronomy, Inc., under NASA contract NAS 5-26555.

\section{References}

1. B. Balick, J. Wilson, A.R. Hajian: AJ 121, 354 (2001)

2. N. Bartel, et al.: Science 287, 112 (2000)

3. E. Berger, et al.: GCN No. 1266 (2002) 
4. E. Berger, S. R. Kulkarni, R. A. Chevalier: ApJ 577, L5 (2002)

5. R.A. Chevalier: ApJ 258, 790 (1982)

6. R.A. Chevalier: ApJ 285, L63 (1984)

7. R.A. Chevalier: ApJ 499, 810 (1998)

8. R.A. Chevalier, C. Fransson: ApJ 420, 268 (1994)

9. R.A. Chevalier, C. Fransson: In Supernovae and Gamma-Ray Bursts. ed. by K. W. Weiler, (Springer, Berlin 2002) in press (astro-ph/0110060)

10. R.A. Chevalier, J.M. Blondin, R.T. Emmering: ApJ 392, 118 (1992)

11. N.N. Chugai: MNRAS 326, 1448 (2001)

12. E. DiCarlo, et al.: ApJ 573, 144 (2002)

13. E. Dwek: ApJ 274, 175 (1983)

14. E. Dwek: ApJ 322, 812 (1987)

15. A. Fassia, et al.: MNRAS 318, 1093 (2000)

16. A. Fassia, et al.: MNRAS 325, 907 (2001)

17. A.V. Filippenko: AJ 97, 726 (1989)

18. D.W. Fox, et al. MNRAS 319, 1154 (2000)

19. C. Fransson, C.-I. Björnsson: ApJ 509, 861 (1998)

20. C. Fransson, P. Lundqvist, R.A. Chevalier: ApJ 461, 993 (1996)

21. C. Fransson, et al.: ApJ 572, 350 (2002)

22. T.J. Galama, et al.: Nature 395, 670 (1998)

23. C.L. Gerardy, et al.: ApJ 575, 1007 (2002)

24. J.C. Houck, J.N. Bregman, R.A. Chevalier, K. Tomisaka: ApJ 493, 431 (1998)

25. K. Hurley, et al.: GCN No. 1252 (2002)

26. S. Immler, S., W. H. G. Lewin: In Supernovae and Gamma-Ray Bursts. ed. by K. W. Weiler, (Springer, Berlin 2002) in press (astro-ph/0202231)

27. S. Immler, W. Pietsch, B. Aschenbach: A\&A 331, 601 (1998)

28. S. Immler, B. Aschenbach, Q. D. Wang: ApJ 561, L107 (2001)

29. S. Immler, A. S., Wilson, Y. Terashima: ApJ 573, L27 (2002)

30. K. Iwamoto, K. Nomoto, P. Höflich, H. Yamaoka, S. Kumagai, T. Shigeyama: ApJ 437, L115 (1994)

31. K. Iwamoto, et al.: Nature 395, 672 (1998)

32. B. Jun, T.W. Jones, M.L. Norman: ApJ 468, L59 (1996)

33. S.R. Kulkarni, et al.: Nature 395, 663 (1998)

34. D.C. Leonard, A.V. Filippenko, A.J. Barth, T. Matheson: ApJ 536, 239 (2000)

35. Z.-Y. Li, R. A. Chevalier: ApJ 526, 716 (1999)

36. Q.-Z. Liu, J.-Y. Hu, H.-R. Hang, Y.-L. Qiu, Z.-X. Zhu, Q.-Y. Qiao: A\&A 144, 219 (2000)

37. J.M. Marcaide, et al.: Science 270, 1475 (1995)

38. J.M. Marcaide, et al.: ApJ 486, L31 (1997)

39. T. Matheson, A.V. Filippenko, L.C. Ho, A.J. Barth, D.C. Leonard: AJ 120, 1499 (2000)

40. C.D. Matzner, C.F. McKee: ApJ 510, 379 (1999)

41. N. Mauron, P.J. Huggins: A\&A 359, 707 (2000)

42. P.A. Mazzali, et al.: ApJ 572, L61 (2002)

43. E. Michael, et al.: ApJ 574, 166 (2002)

44. A.J. Mioduszewski, V.V. Dwarkadas, L. Ball: ApJ 562, 869 (2001)

45. K. Nomoto, T. Suzuki, T., Shigeyama, S. Kumagai, H. Yamaoka, H. Sato: Nature 364, 507 (1993)

46. D. Pooley, et al.: ApJ 572, 932 (2002)

47. P. Rodriguez Pascual, et al.: IAUC No. 7821 (2002) 
48. S. Ryder, K. Kranz, E. Sadler, R. Subrahmanyan: IAUC No. 7777 (2001)

49. G. Schaller, D. Schaerer, G. Meynet, A. Maeder: A\&AS, 96, 269 (1992)

50. E.M. Schlegel: ApJ 556, L25 (2001)

51. S.J. Smartt, G.F. Gilmore, C.A. Tout, S.T. Hodgkin: ApJ, 565, 1089 (2002)

52. C.J. Stockdale, et al.: IAUC No. 7830 (2002)

53. F.K. Sutaria, P. Chandra, S. Bhatnagar, A. Ray: A\&A, submitted (astro-ph/0207137) (2002)

54. D.A. Swartz, K.K. Ghosh, M.L. McCollough, T.G. Pannuti, A.F. Tennant, K.Wu: ApJ, submitted (astro-ph/0206160) (2002)

55. S. Uno, et al.: ApJ 565, 419 (2002)

56. S.D. van Dyk, R.A. Sramek, K.W. Weiler, N. Panagia: ApJ 409, 162 (1993)

57. S.D. van Dyk, K.W. Weiler, R.A. Sramek, M.P. Rupen, N. Panagia: ApJ 432, L115 (1994)

58. K.W. Weiler, N. Panagia, M. J. Marcos, R.A. Sramek: ARA\&A 40, 387 (2002)

59. S. Wellstein, N. Langer: A\&A 350, 148 (1999)

60. S.E. Woosley, R.G. Eastman, T.A. Weaver, P. A. Pinto: ApJ 429, 300 (1994)

61. S.E. Woosley, R.G. Eastman, B.P. Schmidt: ApJ 516, 788 (1999)

62. T.R. Young, E. Baron, D. Branch: ApJ 449, L51 (1995) 